\title{
QUALIDADE DE VIDA DE DEFICIENTES VISUAIS
}

\author{
QUALITY OF LIFE OF VISUALLY IMPAIRED PEOPLE
}

Zélia Z L C Bittencourt ${ }^{1}$, Eduardo Luiz Hoehne²

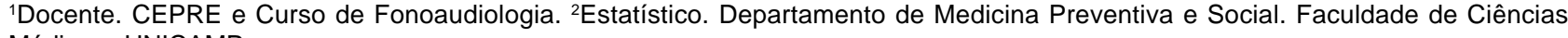 \\ Médicas - UNICAMP \\ CorrespondênCIA: Profa Dra Zélia Z L C Bittencourt. Rua José Morano, 506. CEP 13100-055 - Campinas - São Paulo \\ Telefone: 19 3252-5655 - Fax: 01937888814 \\ E-mail: zeliaz @ fcm.unicamp.br
}

Bittencourt ZZLC, Hoehne EL. Qualidade de vida de deficientes visuais. Medicina (Ribeirão Preto) 2006; 39 (2): 260-264.

RESUMO: Estudo exploratório analítico. Objetivo: Avaliar a qualidade de vida de deficientes visuais em Programa de Reabilitação de um serviço universitário. Métodos: O estudo foi realizado nos meses de outubro e novembro de 2004, com 16 deficientes visuais adolescentes e adultos acompanhados em programa de reabilitação, dos quais 10 tinham baixa visão e 6 eram cegos. Para a avaliação da qualidade de vida foi utilizada a versão em português do World Health Organization Quality of Life (WHOQOL- Bref). Na análise estatística utilizou-se o programa SPSS com escores transformados de quatro a 20. As medianas dos escores de cada domínio foram comparadas e consideradas significativas se "p" fosse menor ou igual a $5 \%$. O estudo foi aprovado pelo Comitê de Ética em Pesquisa. Resultados: Dos 16 deficientes estudados $50 \%$ eram do sexo masculino, idade variando de 18 a 61 anos, média de 38 anos. A avaliação dos diferentes itens do WHOQOL-Bref não mostrou diferença estatisticamente significante entre os pacientes com baixa visão e os cegos. O estudo revelou melhor qualidade de vida nos domínios psicológico $(15,66)$, e físico $(15,14)$. Nas questões gerais o escore global foi 14,00 . Conclusão: Os resultados apontam para uma boa percepção de qualidade de vida nos aspectos físicos e psicológicos e menor percepção nos domínios das relações sociais e do meio ambiente, provavelmente decorrentes da situação sócio-estruturais e culturais dos pacientes.

Descritores: Qualidade de Vida. Questionário. Baixa Visão. Cegueira. Reabilitação.

\section{1- INTRODUÇÃO}

As questões examinadas nesta pesquisa pertencem ao campo da saúde e dizem respeito à avaliação da qualidade de vida de deficientes visuais usuários de programas de reabilitação em um serviço universitário de referência em Campinas.

A avaliação de qualidade de vida $(\mathrm{QV})$ vem crescendo em importância como medida na avaliação de intervenções terapêuticas, de serviços e da prática assistencial cotidiana na área da saúde ${ }^{1,2}$ Tem sido considerada importante indicador devido ao impacto físico e psicossocial que enfermidades, disfunções ou incapacidades podem acarretar para as pessoas acometidas $^{3}$. A melhoria da qualidade de vida passou a ser um dos resultados esperados das práticas assistenciais e das políticas públicas para o setor no campo da promoção da saúde ${ }^{3}$.

Qualidade de vida foi definida pela Organização Mundial da Saúde como "a percepção do indivíduo de sua posição na vida, no contexto da cultura e do sistema de valores em que vive e em relação aos seus objetivos, expectativas, padrões $e$ percepções" 4,5 . 
A literatura dispõe de inúmeros instrumentos para a avaliação da qualidade de vida, tanto genéricos, que podem ser aplicados a várias situações, tais como o Sickness Impact Profile (SIP), o SF $36^{6}$, e o WHOQOL (World Health Organization Quality of Life $)^{3}$ entre outros, quanto específicos, utilizados para determinada doença ou situação. Vários questionários são utilizados para medir a qualidade de vida de deficientes visuais, dentre os quais o Activities of Daily Vision Scale (ADVS), o Vision Function Questionnaire (VFQ), o Low Vision Quality-of-Life Questionnaire $(\mathrm{LVQOL})^{7}$, concebidos para a avaliação de doenças oculares $^{8}$.

A visão é considerada a grande promotora da integração do indivíduo em atividades motoras, perceptivas e mentais ${ }^{9}$ e a perda da mesma pode provocar marcantes alterações, diminuindo sua capacidade de adaptação na sociedade.

Programas de reabilitação são fundamentais na promoção de saúde para que as pessoas cegas ou com baixa visão possam desenvolver-se e ter uma participação efetiva na sociedade. Este estudo tem como objetivo avaliar alguns aspectos da qualidade de vida de deficientes visuais em Programa de Reabilitação de um serviço universitário.

\section{2- MATERIAL E MÉTODOS}

Foi realizada uma investigação exploratória sobre a qualidade de vida de deficientes visuais adolescentes e adultos em acompanhamento no Centro de Estudos e Pesquisas em Reabilitação Prof. Dr. Gabriel Porto (CEPRE), nos meses de outubro e novembro de 2004. A população estudada foi constituída por uma amostra de 16 deficientes visuais que se dispuseram a participar da investigação. O critério de inclusão no estudo foi ser usuário regular do programa de reabilitação e assinar o termo de consentimento informado. O instrumento utilizado para a avaliação da Qualidade de Vida foi o World Health Organization Quality of Life (WHOQOL-BREF), versão em português composto por 24 questões abrangendo os domínios: físico, psicológico, relações sociais, meio ambiente, além de duas questões gerais ${ }^{3}$. A partir da aplicação do questionário de qualidade de vida, realizou-se um levantamento utilizando-se dados da ficha de informações sobre os respondentes para a caracterização sócio-demográfica dos pacientes. Após a coleta do material, os dados foram tabulados e analisados descritivamente. Cada domínio do WHOQOL foi analisado isolada- mente. Foram estudados os 16 deficientes visuais em conjunto e para a análise avaliou-se separadamente os pacientes cegos $(n=6)$ e os com baixa visão $(n=10)$, comparando-se os escores. O estudo obedeceu à metodologia do instrumento, tendo como base uma escala de valores numéricos de 1 a 5 sendo que para cada resposta correspondia um escore específico. Para fins de análise, as questões 3,4 e 26 tiveram seus escores invertidos em função de $1=5,2=4,3=3,4=2,5=1$ sendo que maiores escores correspondem a melhor qualidade de vida. Foi realizada a análise descritiva de todas as variáveis dos questionários e comparou-se os cegos com os de baixa visão. A comparação foi realizada pelo teste de Mann-Witney (teste nãoparamétrico), e as medianas dos escores de cada domínio foram comparadas e consideradas significativas se $\mathrm{p}$ fosse menor ou igual a $5 \%$. Todas as análise foram executadas por meio do software SPSS, com sintaxe específica do instrumento, tendo os escores transformados de quatro a 20. Este estudo foi aprovado pelo Comitê de Ética em Pesquisa da Faculdade de Ciências Médicas da UNICAMP.

\section{3- RESULTADOS}

\section{Características da amostra}

Observou-se que $50 \%$ dos entrevistados eram do sexo masculino, com idade variando de 18 a 61 anos, sendo a idade média de 38 anos, em fase produtiva. Com relação à escolaridade, $31,25 \%$ tinham o ensino fundamental incompleto, $56,25 \%$ freqüentaram até o ensino médio e 12,50\% concluíram o curso superior. Quanto à procedência, 6 pacientes $(37,50 \%)$ residiam na cidade de Campinas e os demais em outras cidades do Estado de São Paulo. No tocante à situação previdenciária, $68,75 \%$ dependiam de benefícios do INSS, $18,75 \%$ eram dependentes de familiares e somente $6,25 \%$ exerciam atividade profissional regular (Tabela I). Destes deficientes visuais, $62 \%$ não necessitavam de acompanhantes para se locomover.

No que se refere às causas que os levaram a procurar um serviço de reabilitação o glaucoma aparece em $25 \%(n=4)$ dos casos, seguido por trauma devido a acidente de trabalho em $12,5 \%(\mathrm{n}=2)$, e as demais distribuídas igualmente em: atrofia do nervo óptico, retinose pigmentar, doença de Stargart, retinoblastoma, coriorretinite macular, retinopatia diabética, retinopatia da prematuridade, catarata, miopia e descolamento de retina (Tabela II). 
Tabela I: Dados sociodemográficos dos pacientes estudados

$\begin{array}{lcc}\text { Variável } & \boldsymbol{n} & \boldsymbol{\%} \\ \text { Sexo } & & \\ \quad \text { Feminino } & 8 & 50,00 \\ \quad \text { Masculino } & 8 & 50,00 \\ \text { Idade (anos) } & & \\ \quad<20 & 2 & 12,50 \\ 21-40 & 7 & 43,75 \\ 41-60 & 6 & 37,50 \\ >60 & 1 & 6,25\end{array}$

Escolaridade

Ensino fundamental incompleto

$5 \quad 31,25$

Ensino médio incompleto

$4 \quad 25,00$

Ensino médio completo

$5 \quad 31,25$

Superior

$2 \quad 12,50$

Situação Previdenciária

Aposentado

$8 \quad 50,00$

Auxílio Doença

$3 \quad 18,75$

Dependente

$3 \quad 18,75$

Atividade

2

6,25

Procedência

$\begin{array}{lll}\text { Campinas } & 6 & 37,50 \\ \text { Leme } & 3 & 18,75 \\ \text { Outras cidades do estado de SP } & 6 & 43,75\end{array}$

\section{Avaliação da qualidade de vida}

No estudo conjunto dos 16 usuários, ao se analisar as medianas nos vários domínios detectamos que os domínios psicológico e físico alcançaram os melhores escores (15,66 e 15,14 respectivamente), seguindo-se pelas relações sociais $(13,33)$ e por último o meio ambiente $(13,25)$. Nas questões gerais: "Como você avalia sua qualidade de vida? e "Você está satisfeito com sua saúde atual ?" o escore global foi 14,00 (Tabela III).
Tabela II: Causas da deficiência visual

\begin{tabular}{|l|c|c|}
\hline Causas da deficiência & $\boldsymbol{n}$ & $\boldsymbol{\%}$ \\
\hline Glaucoma & 4 & 25,50 \\
\hline Trauma (acidente de trabalho) & 2 & 12,50 \\
\hline Atrofia do nervo optico & 1 & 6,25 \\
\hline Retinose pigmentar & 1 & 6,25 \\
\hline Doença de Stargart & 1 & 6,25 \\
\hline Retinoblastoma & 1 & 6,25 \\
\hline Coriorretinite macular & 1 & 6,25 \\
\hline Retinopatia diabética & 1 & 6,25 \\
\hline Retinopatia prematuridade & 1 & 6,25 \\
\hline Catarata & 1 & 6,25 \\
\hline Descolamento de retina & 1 & 6,25 \\
\hline Miopia & 1 & 6,25 \\
\hline Total & 16 & 100,00 \\
\hline
\end{tabular}

\section{Tabela III - Medianas dos vários domínios do WHOQOL-BREF}

$\begin{array}{lcc}\text { Domínio } & \begin{array}{c}\text { Mediana } \\ (\boldsymbol{n}=16)\end{array} & \text { Desvio padrão } \\ \text { Físico } & 15,14 & 3,17 \\ \text { Psicológico } & 15,66 & 3,02 \\ \text { Relações sociais } & 13,33 & 3,54 \\ \text { Meio ambiente } & 13,25 & 1,99 \\ \text { Questões gerais } & 14,00 & 2,15\end{array}$

Com relação à percepção de qualidade de vida $68,7 \%$ dos entrevistados relataram boa ou muito boa qualidade de vida, $12,5 \%$ nem ruim nem boa e $18,8 \%$ ruim. Da mesma maneira, 62,5\% dos entrevistados estão satisfeitos ou muito satisfeitos com sua saúde e $37,5 \%$ nem satisfeitos nem insatisfeitos.

Ao se comparar os pacientes com baixa visão e os cegos, na análise das medianas, nas pontuações dos domínios específicos e nas questões gerais do questionário não se observou diferença estatisticamente significante entre tais pacientes. $\mathrm{Na}$ análise das me- 
dianas utilizando-se a escala ajustada de 4 a 20, cujo ponto médio é 12 , foi possível verificar que as medianas encontram-se acima deste valor. (Tabela IV).

\begin{tabular}{|c|c|c|c|}
\hline Domínio & $\begin{array}{c}\text { Baixa visão } \\
(n=10)\end{array}$ & $\begin{array}{c}\text { Cegueira } \\
(n=6)\end{array}$ & $p$ \\
\hline Físico & 13,42 & 15,14 & 0,6223 \\
\hline Psicológico & 16,00 & 15,33 & 0,6622 \\
\hline Relações sociais & 14,66 & 13,33 & 0,5788 \\
\hline Meio ambiente & 13,00 & 13,25 & 0,4433 \\
\hline Questões gerais & 14,00 & 15,00 & 0,9097 \\
\hline
\end{tabular}

\section{4- DISCUSSÃO}

Apesar de vários estudos sobre Qualidade de Vida de deficientes visuais, cegos ou com baixa visão, na literatura há poucas investigações que enfoquem a qualidade de vida em sujeitos em programas de reabilitação. Neste estudo, foi utilizado para a avaliação da qualidade de vida o instrumento genérico WHOQOL - Bref, que vem comprovar de forma estatística, que o fato de ser cego ou ter baixa visão não apontou diferença significativa entre os dois grupos. Possivelmente, há outros fatores influenciando neste resultado, dentre eles o fato de estarem em processo de reabilitação. Observou-se escores mais altos nos domínios psicológico e físico, enquanto os domínios das relações sociais e do meio ambiente, foram os mais prejudicados pois levam em conta aspectos estruturais como a situação sócio-econômica e cultural do sujeito.

O Centro de Estudos e Pesquisas em Reabilitação Prof. Dr. Gabriel Porto (CEPRE) da Universidade Estadual de Campinas é conveniado ao Sistema Único de Saúde (SUS) e conta com equipe multidisciplinar de professores especialistas que atuam em diversas áreas: serviço social, terapia ocupacional, pedagogia, atividades de vida diária, psicologia, informática e Braille, em Programa de Reabilitação a deficientes visuais, cegos ou com baixa visão. A equipe atua com tecnologia e recursos necessários para implementar a independência e a inclusão social das pessoas deficientes visuais.

O processo de reabilitação implica ações interdisciplinares educativas que valorizam a independência e autonomia do paciente, buscando a redução das per- das funcionais, possibilitando o acesso a informações e conhecimentos com vista à promoção da saúde.

Contraditoriamente, no Brasil, a situação de doença ou de deficiência é para muitas pessoas a possibilidade de inclusão na sociedade, saindo do isolamento e da vulnerabilidade de seu entorno familiar e social. A equipe de saúde ao identificar as necessidades e as condições do meio sociocultural dos usuários, busca ações que visem aumentar a capacidade do indivíduo de tomar decisões neste processo que inclui as dimensões intelectuais, psicológicas e sociais ${ }^{10}$.

A escolaridade é fator importante no processo de reabilitação, uma vez que o aproveitamento do resíduo visual de pessoas com baixa visão está ligado ao contexto social e a independência necessária para a melhoria da qualidade de vida. Em países desenvolvidos a população no geral ocupa-se de leitura e escrita entre suas atividades diárias, demandando um nível de acuidade visual compatível. No que diz respeito à escolaridade $68,7 \%$ dos pacientes do grupo estudado, tinham o ensino médio e superior, e a utilização de ajudas óticas pode proporcionar benefícios pessoais e sociais, aumentando a autonomia e melhorando a qualidade de vida. ${ }^{11}$

A população estudada não apresentou diferença significativa em relação ao sexo, conforme já descrito na literatura. ${ }^{12}$

O glaucoma, causa prevalente de deficiência visual entre nossos usuários, pela alta frequiência de incapacidade que acarreta é considerada um problema de saúde pública ${ }^{13}$ e situa-se entre as principais causas de cegueira em população idosa ${ }^{10}$. Neste estudo em $75 \%$ dos casos, acometeu mulheres (baixa visão), na faixa etária de 39 a 60 anos e que exerciam atividades no lar.

$\mathrm{Na}$ população estudada, a segunda causa de procura por reabilitação foi o trauma. Traumatismos oculares são responsáveis por significativa procura aos serviços de Oftalmologia, pois causam alteração ocular funcional substancial podendo até levar à cegueira, com prejuízos pessoais, sociais e econômicos importantes ${ }^{14}$. Estes indivíduos cegos por traumatismo devido a acidente de trabalho, são do sexo masculino, na faixa etária entre 45 a 60 anos, em concordância com a epidemiologia do trauma ocular, refletindo a maior exposição do homem a atividades profissionais ${ }^{15}$. Tais sujeitos exerciam atividades profissionais de motorista e de mecânico. Cabe ainda ressaltar a importância socioeconômica dos traumatismos oculares graves para o sistema previdenciário e também para outros setores da economia pela incapacidade funcional temporária ou permanente ${ }^{16}$. 


\section{5- CONCLUSÃO}

Os resultados apresentados mostram uma menor percepção de qualidade de vida nos domínios das relações sociais e de meio ambiente, decorrentes da situação sócio-estrutural e cultural dos pacientes. A despeito da boa condição física e psicoló- gica as pessoas carecem de apoio de redes sociais para melhoria das condições ambientais, que inclui a moradia, transporte, etc. Sugere-se a utilização do WHOQOL - bref na avaliação da qualidade de vida em deficientes visuais antes do inicio do processo de reabilitação e a posterior comparação, para confirmar estes achados.

Bittencourt ZZLC, Hoehne EL. Quality of life of visually impaired people. Medicina (Ribeirão Preto) 2006; 39 (2): 260-264.

ABSTRACT: Design of the study: Analytic/Exploratory survey. Purpose: To assess the quality of life of visually impaired people who attend the rehabilitation program at a universitary service. Methods: This study was conducted in October and November 2004. Were interviewed sixteen visually impaired teenagers and adults who attended the rehabilitation program: blind $(n=10)$ and low vision $(n=6)$. In order to assess their quality of life, we used the Portuguese version of the World Health Organization Quality of Life (WHOQOL - Bref). SPSS was used and the scores were transformed from 4 to 20. The medians of the scores in each domain were compared and considered significant when $p=0,05(5 \%)$. The Research Ethics Committee approved this investigation. Results: The population of this research consisted of $50 \%$ men, aged from 18 to 61 years, with an average of 38 years of age. The evaluation of the different items of the WHOQOL Bref did not indicate a statistically significant difference between low vision and blind patients. The study pointed out a better quality of life in the psychological and physical aspects $(15,66$ and 15,14 , respectively). In the general questions the global score was 14,00 . Conclusion: The results reported a good quality of life perception in the physical and psychological domains and a less perception in the social relationship and environment.

Keywords: Quality of Life. Questionnaire. Low Vision. Blindness. Rehabilitation.

\section{REFERÊNCIAS}

1 - Bittencourt ZZLC, Alves Filho G, Mazzalli M, Santos NR. Qualidade de vida em transplantados renais: importância do enxerto funcionante. Rev Saúde Pública 2004; 38: 732-4.

2 - Minayo MCS, Hartz ZMA, Buss PM. Qualidade de vida em saúde: um debate necessário. Ciên Saúde Coletiva 2000; 5:7-18.

3 - Seidl EMF, Zannon CMLC. Qualidade de vida e saúde: aspectos conceituais e metodológicos. Cad Saúde Pública, Rio de Janeiro, 2004; 20(5): 580-8.

4 - Fleck MPA, Fachel O, Louzada S, Xavier M, Chachamovich E, Vieira G, Santos L, Pinzon V. Aplicação da versão em português do instrumento abreviado de avaliação de qualidade de vida WHOQOL-bref. Rev Saúde Pública 2000; 34: 178-83.

5 - Whoqol Group. The World Health Organization quality of life assessment (WHOQOL): position paper from the World Health Organization. Soc Sci Méd 1995; 10:1403-9.

6 - Ciconelli RM, Ferraz MB, Santos W, Meinão I, Quaresma MR. Tradução para a língua portuguesa e validação do questionário genérico de avaliação de qualidade de vida SF - 36 (Brasil SF-36). Rev Brasil Reumatol 1999; 39: 143-50.

7 - Wolffsohn J, Cochrane A. Design of low vision quality-of-life questionnaire (LVQOL) and measuring the outcome of lowvision rehabilitation. Am J Ophthalm 2000; 130: 793-802.

8 - Ferraz EVAP, Lima CA, Celia W, Arieta CEL. Adaptação de questionário de avaliação da qualidade de vida para aplicação em portadores de catarata. Arq Bras Oftalmol 2002; 65:293-8
9 - Lucas MB, Leal DB, Tavares SS, Barros EA, Aranha ST. Condutas reabilitacionais em pacientes com baixa visão Arq Bras Oftalmol 2003; 66: 77-82.

10 - Temporini ER, Kara-José N, Gondim EL, Dantas FJ. Conhecimentos sobre saúde ocular entre profissionais de um Hospital Universitário. Medicina, Ribeirão Preto, 2002; 35: 53-61.

11 - Carvalho K M, Monteiro GBM, Isaac CR, Shiroma LO, Amaral MS. Causes of low vision and use of optical aids in the elderly. Rev Hosp Clin Fac Méd Univ São Paulo, 2004; 59: 157-60.

12 - Aragaki GN, Inada ET, Teixeira MF, Almeida JR GC, Kashiwabuchi LK. Epidemiological study of severe ocular trauma in a University Hospital of São Jose do Rio Preto SP. Arq Bras Oftalmol 2003; 66: 473-6.

13 - Oliveira A, Paranhos JR A, Prata JR JA. Características dos pacientes atendidos pela primeira vez no setor de glaucoma da Universidade Federal de São Paulo - UNIFESP. Arq Bras Oftalmol 2003; 66: 785-90.

14 - Bordon AF, Souza LB, Moraes NSB, Freitas D. Perfuração ocular: estudo de 473 casos. Arq Bras Oftalmol 1994; 57: 62-5.

15 - Molinari LC.Trauma ocular e endoftalmite. Rev Bras Oftalmol 1995; 54: 775-81.

16 - Alves MR, Kara-Jose, N. O trauma ocular como causa de cegueira. Rev Med (São Paulo) 1997;76: 297-302.

Recebido em 28/11/2005

Aprovado em 25/04/2006 